\title{
Service Delivery Platform for Open IPTV Convergence Service Creation and Management
}

\author{
Changwoo Yoon \\ ETRI (Electronics and Telecommunications Research Institute), Korea \\ cwyoon@etri.re.kr
}

\begin{abstract}
IPTV brought a new digital era in the broadcasting domain. The main change of digital broadcasting is an advent of a bi-directional interactive service feature. Using that bidirectionality, IPTV provides various kinds of convergence service such as information providing, advertisement, and ecommerce. But the number of services required to be developed and to be provided to users geometrically increases and the lifetime of services is shortened requiring services to be developed within a short period. To support the market demand for a massive service delivery, the IPTV platform based on service delivery platform (SDP) is essential. The SDP is a technique developed to meet the variation in communication and information technology convergence environments, allows service providers to rapidly create and deliver convergence services. This paper describes an Open IPTV service delivery platform structure and method for supporting rapid service creation by reusing pre-defined IPTV service components. We describe key components and functionalities of SDP, IPTV Enablers and its combination for service convergence. We show customer targeted mobile advertisement for Open IPTV convergence service example.
\end{abstract}

\section{Keywords-IPTV, SDP, SOA, ACAP, Open IPTV Platform}

\section{INTRODUCTION}

The main environmental change brought on by digital broadcasting such as IPTV, Smart TV and digital cable TV is an advent of a bi-directional broadcasting business that uses a telecommunication return channel. This is a change from 'TV as a medium of transaction', which is one way watching of analogue TV, to 'TV as a marketplace', which connects the viewer directly, collects the viewer's opinion bi-directionally, and reapplies the viewer's reaction to service. Figure 1 shows various examples of bi-directional convergence services combining broadcasting, telecommunication and related information [1,2, and 3].

Using those bi-directional characteristic, IPTV provides various kinds of convergence service such as information providing, advertisement, and e-commerce. But development of new convergence service is difficult because most of IPTV broadcasting system in Korea uses ACAP (Advanced Common Application Platform) as a base middleware platform [4]. The ACAP API has different shape according to IPTV service providers. General Service developer cannot easily develop those telco-dependent ACAP applications.
The characteristic of IPTV convergence service is a dependency with broadcasting content. The convergence services provided during channel service are tightly coupled with the contents of channel service. For example, channel content related information is shown on TV for the information providing service. For the advertisement, content related advertisements are selected and shown on TV.
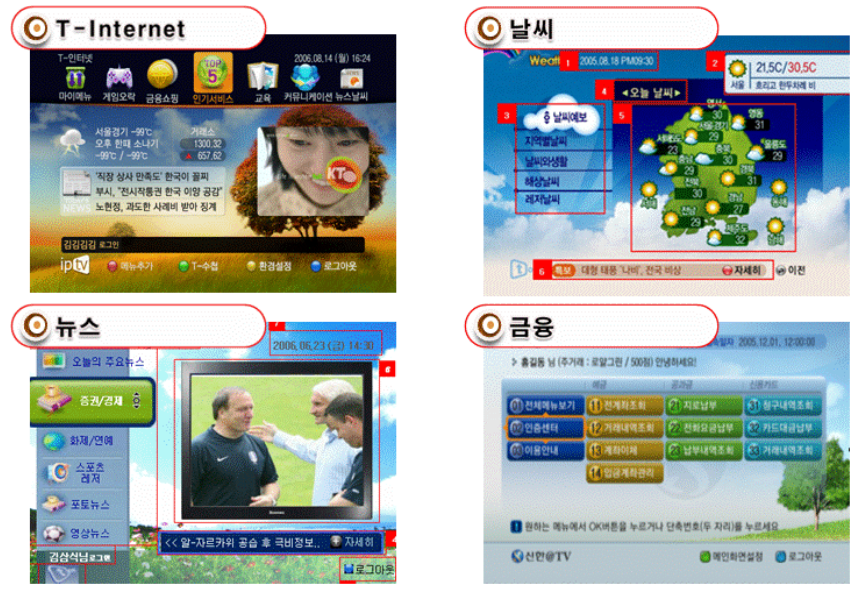

Figure 1. Example of IPTV convergence service

The number of services required to be developed and to be provided to users geometrically increases and the lifetime of services is shortened requiring services to be developed within a short period. Furthermore, with the rapid development of information technology such as web technology, there is a need to integrate information, communication and broadcasting technologies to create new convergence services. Moreover, users want to receive services only for themselves and act not only as service consumers but also service prosumers with the spread of various personal devices. [5] The ACAP is not sufficient to support those requirements.

In the enterprise domain, the SOA (Service Oriented Architecture) has been used for integrating various kinds of business services. In SOA, systems are divided into basic service components. These services are distinct units that can be independently reusable. The Service Delivery Platform (SDP) is one of implementations of the SOA concept in an enterprise domain [6].

A service delivery platform (SDP) is a technique developed to meet this variation in communication and 
information technology environments, allows common carriers to rapidly create and deliver services efficiently to provide the services and assists third party service providers or personal information providers in efficiently participating in service business. [5, 6]

Conventional techniques relating to the SDP mostly define the SDP as a set of enablers corresponding to abstract forms of physical devices of a network or the Internet. These conventional techniques provide general SDP structures in which service common functions such as an operation support system (OSS)/business support system (BSS) are connected with the enablers of the SDP to enable rapid service creation and third party service providers and users can abstract functions to create and provide services even if the third party service providers and the users are not network operators. [7, $8,9]$

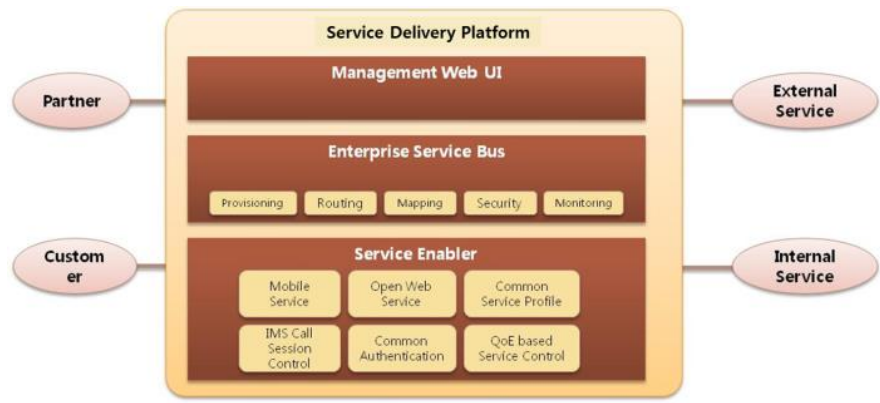

Figure 2. Major charchteristics of SDP

This paper describes an Open IPTV service delivery platform structure and method for supporting circumstances in which users can be a service provider. We describe key components and functionalities of SDP, IPTV Enablers and its combination for service convergence. We show customer targeted mobile advertisement for Open IPTV convergence service example.

\section{KEY COMPONENTS OF SDP}

\section{A. Common BUS}

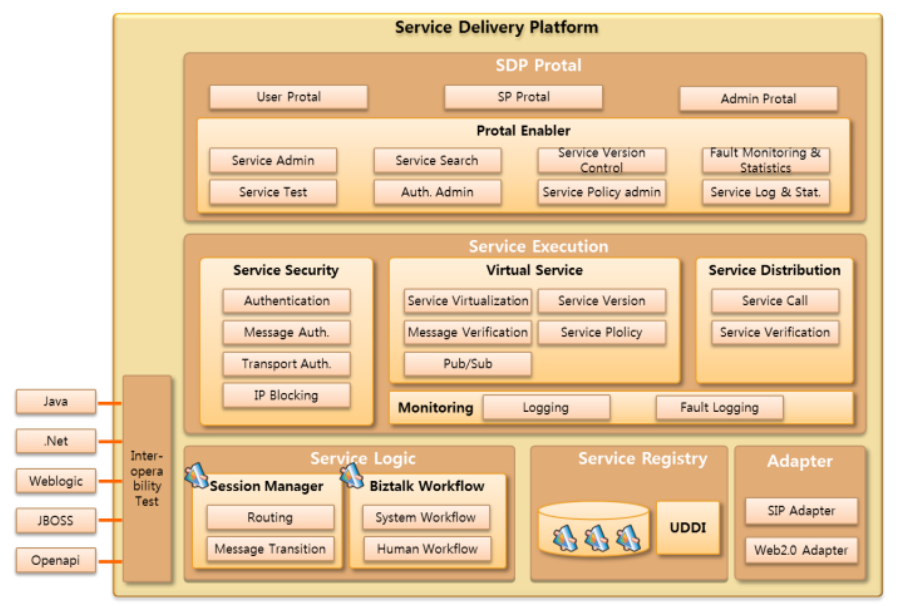

Figure 3. Architecture of SDP
Figure 3 is a common architecture of SDP providing convergence services. The concept of SDP consists of two parts: Common BUS and Enablers. The Common BUS is an execution environment of Web services and web service directories. The common BUS has a similar role with that of the Enterprise Service Bus (ESB). The ESB is a mediating hub at the time of Web Service coordination. It acts as a message broker between services. When an application tries to find Web Service in its routine, ESB finds the appropriate Web Service with application's context.

\section{B. Enablers}

The original concept of enablers came from Open Mobile Alliance (OMA). The enablers are the function that abstracts network functions and provides them to third party application developers through application programming interface (API). Enablers interact with each other and create other services. The OMA does not specify a protocol for the interfaces of enablers or a specific mechanism for combining these enablers. [6] Enabler is a building block that encapsulates reusable functionality.

We used Web services specification for the implementation of enablers. Web Service Description Language (WSDL) is used to describe the interface of services provided by enablers. Simple Object Access Protocol (SOAP) protocol is used to communicate between services.

\section{III.KEY FUNCTIONALITIES OF SDP}

\section{A. Service Virtualization}

Service virtualization is a recent trend of SOA focusing on providing common infrastructure to create and manage complex service eco-system. By using service virtualization, service developer can focus on service feature developing without worrying on how functions are provided, consumed, and managed.

Service virtualization does not modify general service code, rather makes virtual service providing the API functions before executing service. The key idea of service virtualization is service brokering residing between service client and service implementation.

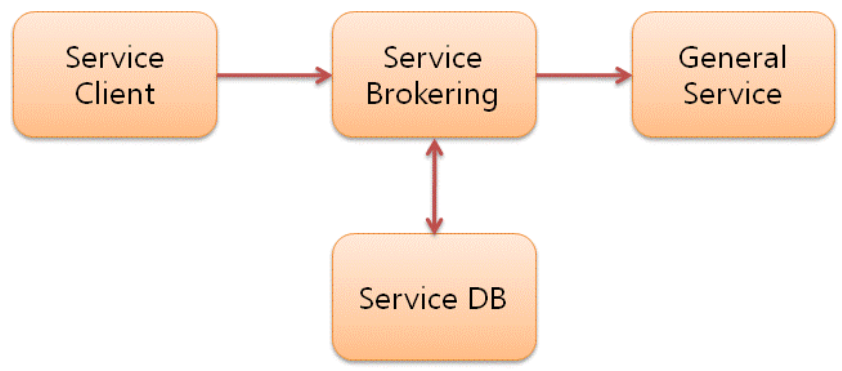

Figure 4. Concept of Service Virtualization

Service brokering is needed for a separation of client and general service. In this architecture, client cannot connect directly with general service. It can only communicate through brokering service. At the position of service brokering, virtualized service is hosted and exported to service user. 
Service broker reads information for DB and decides how to host virtualized service. This approach exports several virtualized service for one general service, we can adopt easily to various customer scenarios.

All communications are transmitted through service broker. We can provide various kinds of brokering services: version control, protocol transfer, monitoring, and real time policy decision. Service virtualization does not change client and general service code.

Service virtualization has several advantages. It provides independent ownership of SOA's each layer. For example, service provider develops service interfaces and logics. Operator describes endpoint policy and message standards. Architect professionals define service level policy and execution patterns. Business owner describes business policy and requirements. All of above can cooperate at the centre points.

Eventually, service virtualization reduces time-to-market for a new service and provides more flexible and substantial methods for managing service environment.

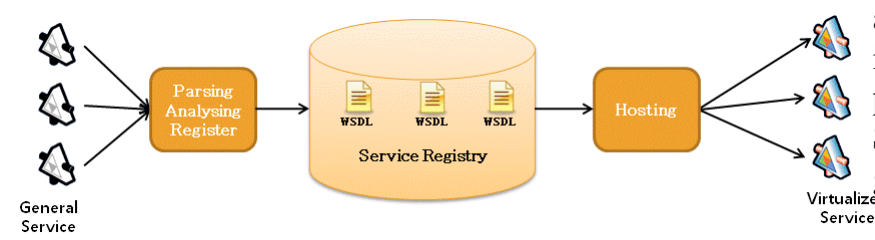

Figure 5. Architecture of Service Virtualization

Figure 5 shows implementation model of service virtualization. It parses general service's WSDL, analyses information, and then registers into service registry.

\section{B. Location Transparency}

For a client, there must be no distinction between the usage of local and remote SOA services. They should be accessed in the same manner as if they were present in the local SOA framework. To provide location transparency, the SDP manages service location at registry and mediate it. With this feature, service execution is not affected by the change of service location.

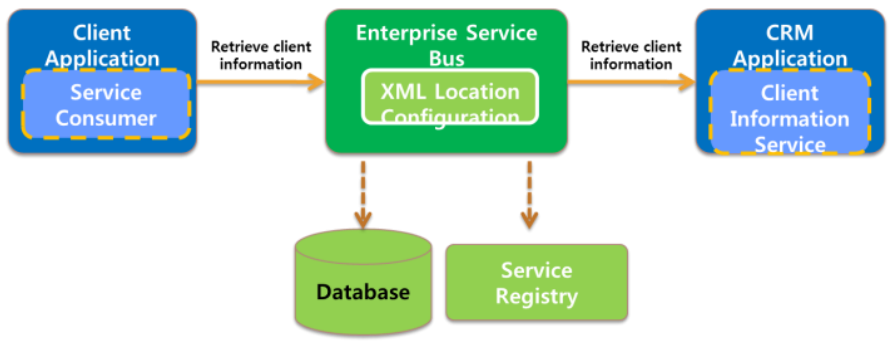

Figure 6. Concept of Location Transparency

\section{Service Authorization}

The SDP service delivery control function assists managing and selling of the value-added services. Delivery control may include user and partner authentication, authorization, credit control, and SLA enforcement. [12]
For services requiring a user limitation, certification is granted an access to only authorized users. Also the SDP authorization function provides WS-Security standard that is Web service authentication capability

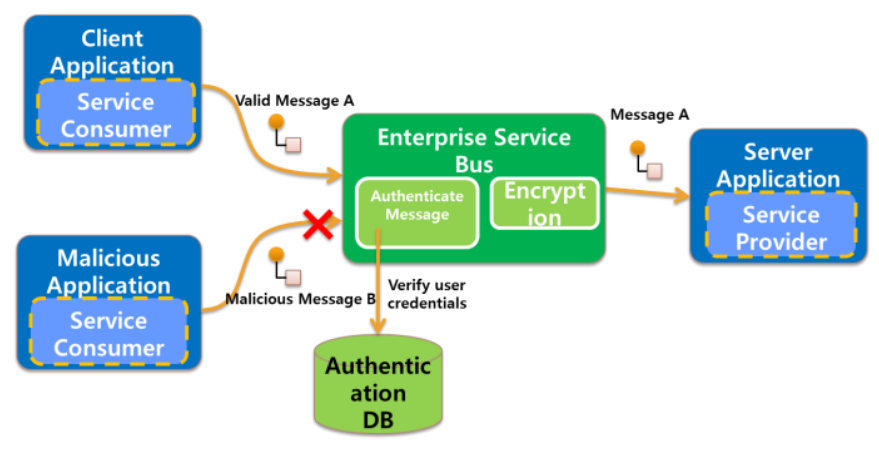

Figure 7. Concept of Service Authorization

\section{Service Protocol Conversion}

To easy the creation of convergence service, an ESB used as a transitional gateway to convert protocols. Today with introduction of WEB 2.0, there are a lot of de-fecto standard protocol like XML/HTTP RPC, REST,JSON etc. Sometimes SOA systems have to support these kinds of protocols. The generic proxy pattern provides a protocol converter.

There are several types of proxy listed below: [13]

- Edge Proxy (Inbound): This is entry point of our ESB. It covers protocol conversion to internal SOA protocol. (Web services)

- Common Proxy: This is point to handle cross cutting concerns for system authorization, authentication, logging, billing etc. And route the service request to appropriate local proxy.

- Local Proxy: It is one-to-one mapping to Business service. It can just forward request to business service or it contains mediation logics. (Intermediary logic Transformation, Routing, Function adding, etc.)

- Edge Proxy (Outbound/Optional): This proxy is another edge proxy for outbound protocol conversion. This is optional. In our architecture internal protocols are integrated with web service. This proxy is used for legacy integration.

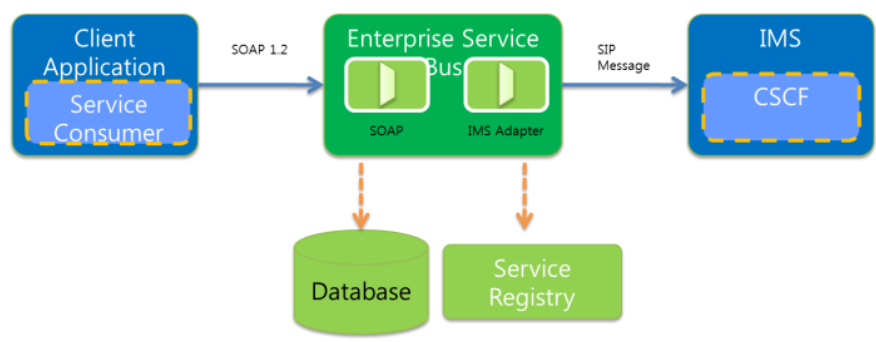

Figure 8. Concept of Service Protocol Translation

\section{E. Message Format Conversion}

Along with the protocol conversion, the message format conversion is needed because the number and types of arguments are different. 


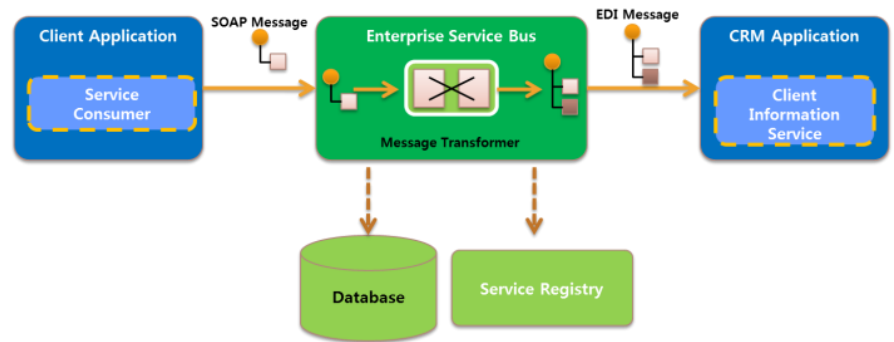

Figure 9. Concept of Message Format Change

\section{F. Message Routing}

Based on the type of individual message, the message routing function sends the message to one of the many services available out-of-the-box on the platform.

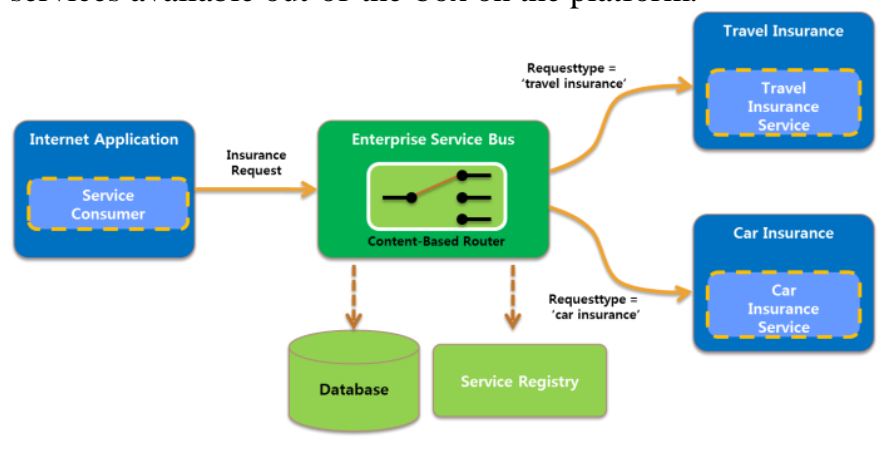

Figure 10. Concept of Message Routing

\section{IV.OPEN IPTV PLATFORM}

IPTV is defined as multimedia services such as television /video/audio/text/graphics/data delivered over IP based networks managed to provide the required level of QoS/QoE, security, interactivity and reliability by ITU-T Focus Group.

If we focus convergence, we can define IPTV as multimedia services delivered over IP based wire \& wireless converged networks managed to provide the required level of QoS/QoE, security, interactivity, reliability and openness, and for any contents to be optimally consumed and generated by users anywhere, anytime, and with any device.

The main features of IPTV are bi-directionality and openness. IPTV platform has an importance to support these features.

Typically, IPTV platform means middleware. The IPTV middleware uses client/server architecture and the client often resides at the STB. The middleware controls the customer interaction with the service and, therefore, plays a very important role in controlling the customer's overall experience. The definition of IPTV middleware is a layer of software between applications and resources, which consists of a set of service enablers that allow multiple functionalities running on one or more devices in an IPTV system to interact across a network. Figure 11 shows a concept of IPTV middleware.

IPTV middleware also defines and manages subscribers, the services available to them, the business rules and the billable transactions associated with their use of the system. It also oversees or directly manages content assets, physical assets, and to an extent, oversees and manages many of the subsystems of the end-to-end IPTV deployment ecosystem. As such, it is extremely complex, not to mention the fact that the operators deploying it are generally not familiar with some of its core enabling technologies, nor with the services it enables.

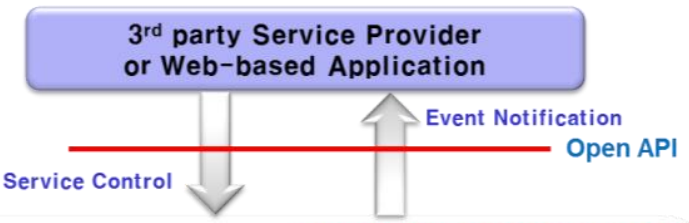

Open Media Service Platform

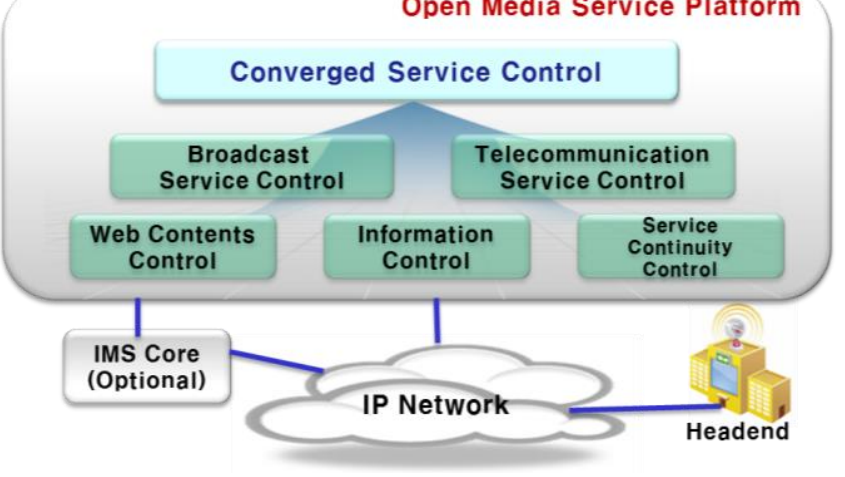

Figure 11.

IPTV middleware concept

Previously, when only wired and wireless telephones existed in the communication environment, network operators or service providers generated and provided services, and the number of generated services was small. A collective system for executing a service, such as a service providing system or a service/user management system was constructed for the service whenever needed. [11]

In the current service environment in which circuit-based communication is changing to Internet-centric packet-based communication and into which wired and wireless communications are integrated, the number of services required to be developed and provided to users geometrically increases and the lifespan of services is shortened to allow for services being developed within a short period. Furthermore, with the rapid development of information technology such as web technology, there is a need to integrate information, communication and broadcasting technologies to create new convergence services. Moreover, users want to receive services only for themselves and act not only as service consumers but also service prosumers with the growing popularity of various personal devices. [11]

To meet these changing environments, there are several attempts to include service platform technologies into IPTV such as Open API and SDP (Service Delivery Platform).

The Platform makes it possible to develop services network provider independently. It shortens the development period by recycling existing services, which makes it easy to develop various convergence and personalized services. The shorter development time gives CAPEX/OPEX (Capital Expenditure, Operational Expenses) advantages to service provider: fast commercialization, extending business opportunities.

Figure 12 shows Open IPTV platform SDP enablers. It has hierarchical structure. At the very low level of the platform, 
network support enablers control network capability according to service request such as bandwidth.

Primitive service enablers are grouped as broadcast control, telecommunication service control, web content control, and information control. In each group, unit enablers are defined that has an atomic function. Combining these unit enablers, the platform consist convergence service control group layer. The combined enablers defined in highest layer looks like service. The application server (AS) executes customer service that calls platform enablers as a reusable function.

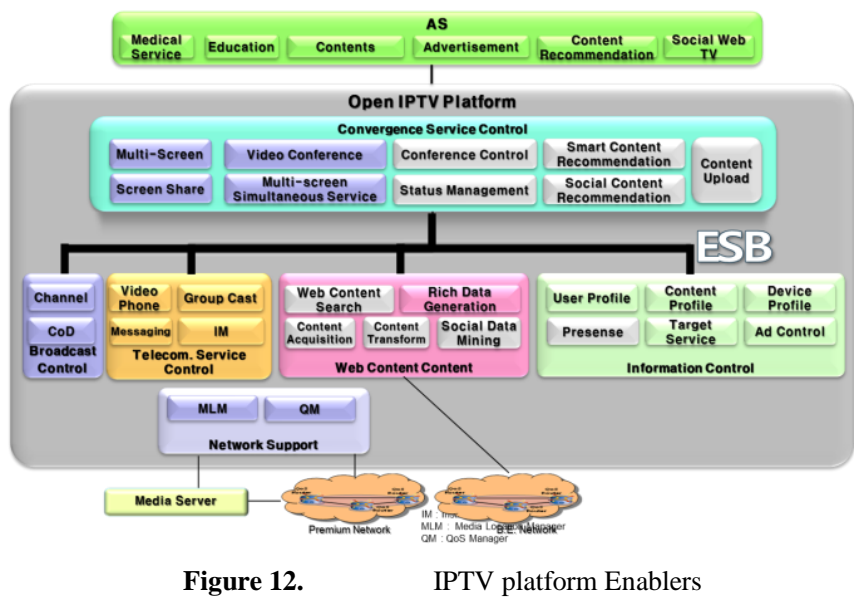

Figure 13 shows how the IPTV service reuses platform enablers. For example, telemedicine service uses group casting, messaging, $\mathrm{CoD}$, presence, and video phone primitive enablers. And it uses screen sharing, simultaneous viewing, conference screen sharing, and conference simultaneous viewing convergence enablers.

Other services such as distance education and smart work shares defined enablers of IPTV platform.

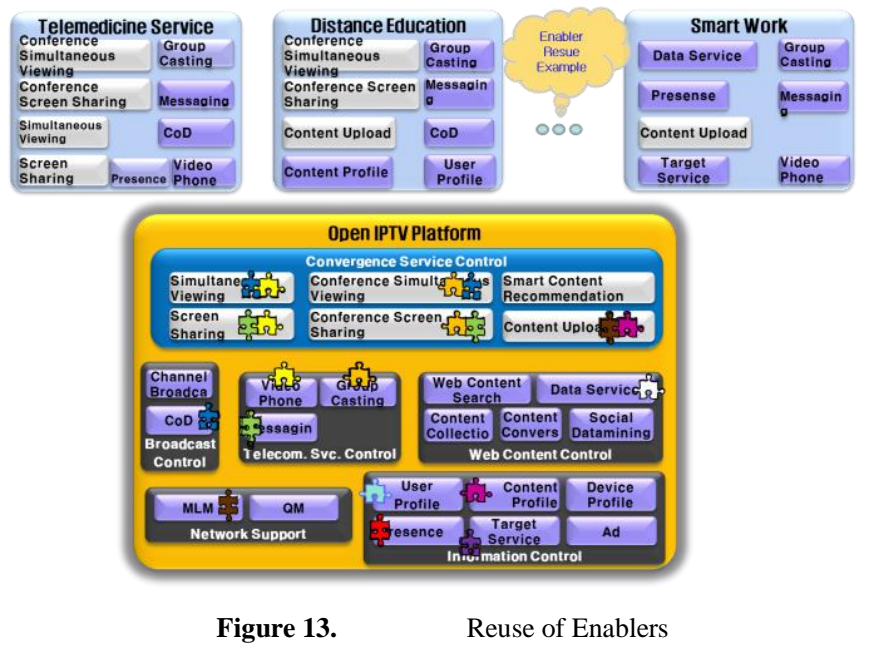

Figure 14 shows targeted advertisement service created using IPTV platform. While customer are watching VOD or real-time channel service, advertisement is sent to user's smartphone that is prepared according to contents and customer properties such as VOD's actor, user's sex, age, etc.

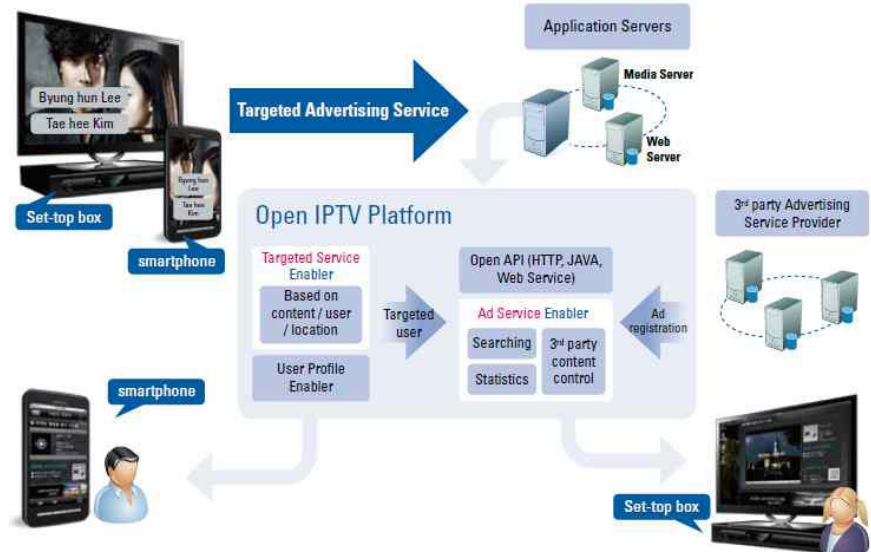

Figure 14. Tatgeted advertisement service

The split EPG terminal has a function controlling IPTV service via home AP. A mobile user watches golf sports channel in VOD service. He already gave his preferences to the profile enabler via open service platform. For instance, since the profile enabler knows that he enjoys golf as outdoor sports, when he detects golf driver on watching golf contents, the mobile advertisement process enabler may give him advertisement moving picture about newly released golf driver. Mobile advertisement process enabler may give helpful information to him as well as connect to the purchase step by the mobile device.

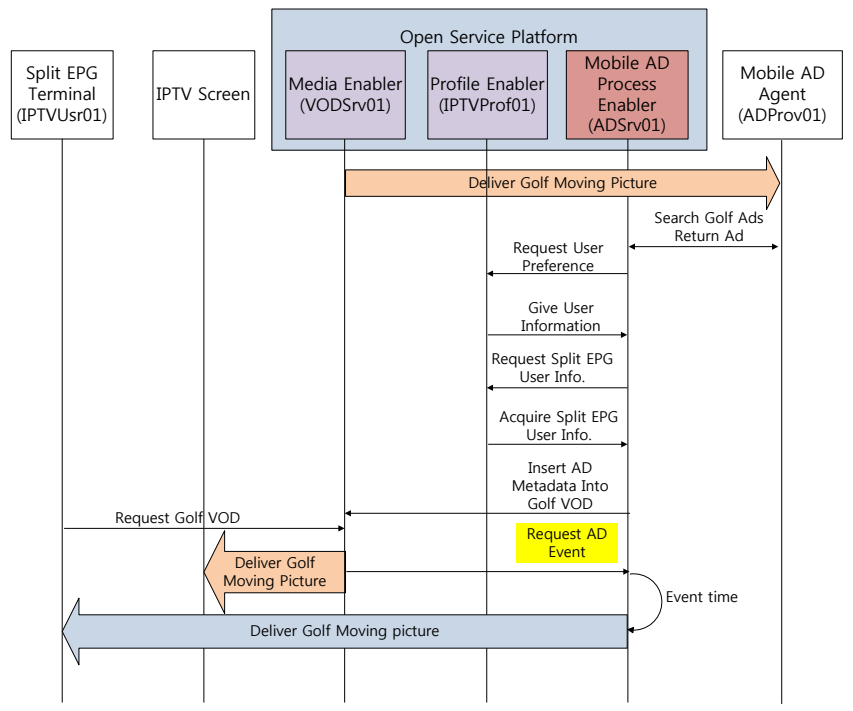

Figure 15. Service flows of Targeted Advertising Service

Fig. 15 shows example of targeted advertisement service using SDP. Mobile advertisement agent may be person or subsystem of an advertisement service provider. He also watches a golf VOD service via media enabler within open service platform. The mobile advertisement process enabler searches a golf advertisement among many advertisement contents of a mobile advertisement agent. That golf advertisement is related with revealed product in golf moving picture. The mobile advertisement process enabler acquires user preference and check if mobile users own split EPG terminals. The 
convergence enabler then inserts advertisement metadata into golf VOD. A mobile user with split EPG terminal requests to view golf contents to open service platform. As soon as golf moving picture is delivered to an IPTV user, advertisement request event is generated.

\section{Conclusions}

Service Delivery Platform (SDP) used for a creation and management of convergence services. Service virtualization is a key concept of SDP for a service convergence because it provides service feature independence among unit services (components) of convergence services.

Service provider can create service components using standard interface without worrying how to integrate each unit services. After creation of service components, the service provider can create convergence service combining those reusable components. Using the SDP's feature, the efforts of service creation can be reduced more than half.

The easy service creation feature will brought a business market chance: creating various business models such as prosumers, service provider, hosting provider, MVNO, and Telco shown in figure 12.

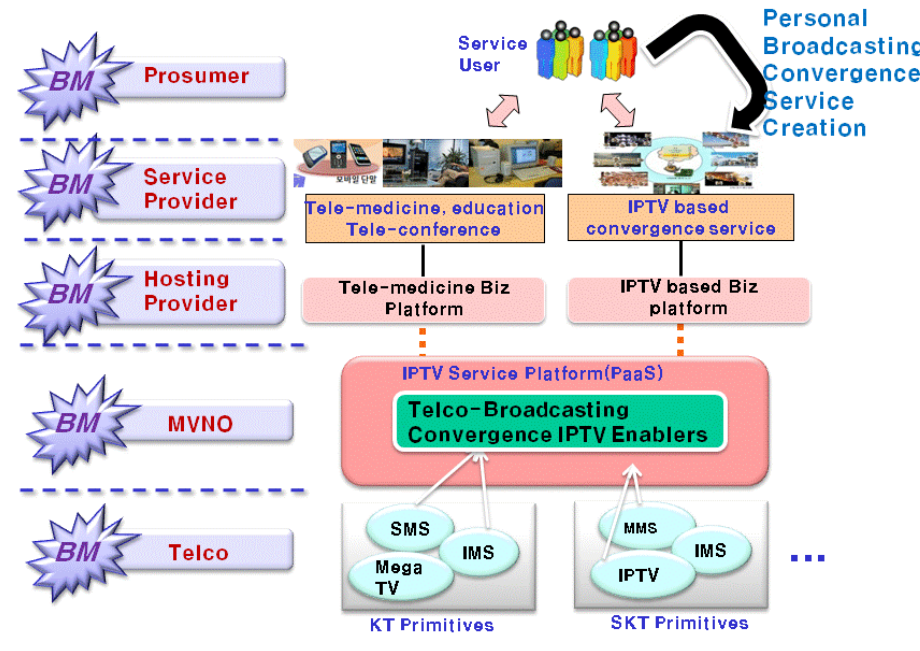

Figure 16.

Business model brought by using SDP

IPTV service is an example of convergence service between telecommunication and broadcasting services. SDP will have an essential role on convergence service creation and management because of its SOA concepts.

We developed several service component groups for SDP based IPTV service platform such as broadcasting service control, telecommunication service control, web contents control, network support, and information control. For example, the telecommunication service control components group consists of video telephone, group casting, messaging.
With those components, IPTV service provider can create convergence IPTV service providing telephone call notice or PIP video-phone screen while watching channel broadcasting.

The SDP expected to be a key part for M2M (Machine to machine) or IoT (Internet of Things) service platform because of its easy way of creating convergence services.

\section{REFERENCES}

[1] Changwoo Yoon, H. Lee, S.H.Jeon, H. Lee, "Mobile Digital Signage System based on Service Delivery Platform," ICT Convergence (ICTC), 2011, pp. 582-586, Sep. 2011

[2] Changwoo Yoon, H. Lee, W. Lyu, B. Kim, "IPTV Service and Evolution direction of technology," KICS magazine, pp. 3-11, Aug 2008

[3] Changwoo Yoon, H.Lee, W.Ryu, "Next Generation IPTV Platform," Optical Internet(COIN) $20109^{\text {th }}$ international conference, pp.1-3, Jul. 2010.

[4] ATSC Standard: Advanced Common Application Platform (ACAP), ww.atsc.org/cms/standards/a_101a.pdf, 2009

[5] Changwoo Yoon, Shinmo Kim, Hyunwoo Lee, "Convergence Service Implementation based on Service Delivery Platform and Research Issues," International Technical Conference on Circuits/Systems, Computers and Communications, pp. 1080-1083, July 2009.

[6] H. Ohnishi, Y. Yamato, M. Kaneko, T. Moriya, M. Hirano, and H Sunaga, "Service delivery platform for teleco-enterprise-Internet combined services", IEEE GLOBECOM, pp.108-112, 2007.

[7] Sun Young Lee, Jong Yun Lee, and Byung Il Lee, "Service Composition Techniques Using Data Mining for Ubiquitous Computing Environments", International Journal of Computer Science and Network Security, Vol 6., No 9B, September 2006.

[8] Nilanjan Banerjee, et. Al., "Telecom Telecom mashups: enabling web 2.0 for telecom services", International Conference on Ubiquitous Information Management and Communication, pp.146-150, 2008.

[9] Moriya, T.; Ohnishi, H.; Yoshida, M.; Hirano, M., "Dataflow Generation for Service Composition to Incorporate Web and Telecommunication", GLOBECOM 2007, pp.26-30, Nov. 2007.

[10] Soonchul Jung Mi-Kyoung Kang Dae-Woo Choi, "Call/Messaging Open API for Telecommunication Services", 10th International Conference on Advanced Communication Technology, pp.1139-1143, Feb. 2008.

[11] Changwoo Yoon, et al., "Convergence Service Implementation based on Service Delivery Platform and Research Issues," ITC-CSCC, 2009

[12] Johnston, Andy, et al. "Evolution of service delivery platforms." Ericsson Review 1 (2007): 19-25.

[13] Terry Cho, "ESB Design Pattern - Generic Proxy Pattern,", http://javamaster.wordpress.com/page/2/, 2009

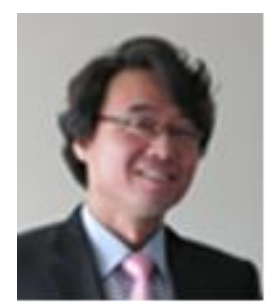

Changwoo Yoon received the B.S. degree from Sogang University, Seoul, Korea, in 1990. He received M.S. degree from POSTECH, Pohang, Korea, in 1992. He received Ph.D. degree in Computer \& Information Science \& Engineering from University of Florida, US, in 2005. Currently he is principal researcher in Creative Service Research team, ETRI and adjunct professor at University of Science and Technology. His current research interests include Future Services, Brain Science, N-Screen, IPTV, Cloud computing, SOA, Service creation/delivery technology and information retrieval. 